\title{
On the Cauchy Problem for Quasilinear Hyperbolic Systems of Partial Differential-Functional Equations of the First Order
}

\author{
T. CZŁAPINSKKI
}

\begin{abstract}
An existence and uniqueness theorem for the generalized solution (in the "almost every where" sense) of the Cauchy problem for a quasilinear functional partial differential system of the first order is proved.
\end{abstract}

Key words: Schauder canonic form, generalized solutions, bicharacteristics

AMS subject classification: $35 \mathrm{~L}$ 50. $35 \mathrm{DOS}$

\section{Introduction}

We denote by $\mathbb{R}^{n}$ the $n$-dimensional real vector space with the norm $\|s\|_{n}=\max _{1 \leq i \leq n}\left|s_{j}\right|$ ( $s=\left(s_{1}, \ldots, s_{n}\right) \in \mathbb{R}^{n}$ ) and by $M(m, k)$ the space of real $m \times k$ matrices. Furthermore by $C(X, Y)$ we denote the usual space of continuous functions from $X$ to $Y$ and by $L_{1}\left(I, \mathbb{R}_{+}\right)$ the usual space of Lebesgue integrable functions, $I \subset \mathbf{R}$ being an interval and $\mathbf{R}_{+}=[0,+\infty)$. Let $J$ be the set of all functions $\varphi=\left(\varphi_{1}, \ldots, \varphi_{m}\right) \in C\left([-h, 0] \times \mathbb{R}^{r}, \mathbb{R}^{m}\right), h \geq 0$, such that, for some $\Lambda, \Gamma \in \mathbb{R}_{+}$and $\omega \in L_{1}\left([-h, 0], \mathbb{R}_{+}\right)$,

$$
\begin{aligned}
& \|\varphi(x, y)\|_{m} \leq \Gamma, \\
& \|\varphi(x, y)-\varphi(x, \bar{y})\|_{m} \leq \Lambda\left\|_{y}-\bar{y}\right\|_{r}, \quad \forall(x, y),(x, \bar{y}),(\bar{x}, y) \in[-h, 0] \times \mathbb{R}^{r} . \\
& \|\varphi(x, y)-\varphi(\bar{x}, y)\|_{m} \leq\left|\int_{x}^{\bar{x}} \omega(t) d t\right|
\end{aligned}
$$

Let $B=\left\{(x, y) \in[-h, 0] \times \mathbb{R}^{r}:\|y\|_{r} \leq b\right\}$, where $0 \leq b \leq+\infty$. If $z \in C\left(B, \mathbb{R}^{m}\right)$, then we write $\|z\|_{B}=\sup \left\{\|z(s, t)\|_{m}:(s, t) \in B\right\}$. We will mean by $K(P, Q)\left(P, Q \in \mathbb{R}_{+}\right)$the set of all functions $w \in C\left(B, \mathbb{R}^{m}\right)$ satisfying the following conditions:

(i) $\|w(s, t)\|_{m} \leq P,\|w(s, t)-w(s, \bar{t})\|_{m} \leq Q\|t-\bar{t}\|_{r} \quad \forall(s, t),(s, \bar{t}) \in B$.

(ii) $\|w(s, t)-w(\bar{s} ; t)\|_{m} s\left|\int_{s}^{s} \tau(\alpha) d \alpha\right|\left(\tau \in L_{1}\left([-h, 0], \mathbb{R}_{+}\right)\right) \forall(s, t),(\bar{s}, t) \in B$. Let $\Omega=\left[0, a_{0}\right] \times \mathbb{R}^{r} \times K(P, Q), \Omega_{0}=\left[0, a_{0}\right] \times \mathbf{R}^{r} \times \mathbb{R}^{m}$, where $a_{0}>0$, and let

$$
A=\left[A_{i j}\right]: \Omega_{0} \rightarrow M(m, m), \rho=\left[\rho_{i j}\right]: \Omega \rightarrow M(m, r), f=\left[f_{1}, \ldots, f_{m}\right]^{+}: \Omega \rightarrow M(m, 1),
$$

where $T$ is the transpose symbol.

For any $a \in\left[0, a_{0}\right]$ let $D_{a}=[0, a] \times \mathbf{R}^{r}, \tilde{D}_{a}=[-h, a] \times \mathbb{R}^{r}$. If $z \in C\left(\widetilde{D}_{a}, \mathbf{R}^{m}\right)$, then for a fixed $(x, y) \in D_{a}$ by $z_{x y}: B \rightarrow \mathbb{R}^{m}$ we denote the function defined by $z_{x y}(s, t)=$ $z(x+s, y+t),(s, t) \in B$.

For $a \in\left(0, a_{0}\right], \varphi \in J$ and some $P, Q \in \mathbb{R}_{+}, \chi \in L_{1}\left(\left[-h, a_{0}\right], \mathbb{R}_{+}\right)$let $K_{a \dot{\varphi}}(P, \chi, Q)$ denote

12 Analysis. Bd. 10, Hefi 2 (1991) 
the set of all functions $z \in C\left(\widetilde{D}_{a}, \mathbb{R}^{m}\right)$ such that

(i) $\|z(x, y)\|_{m} \leq P$,

$$
\begin{array}{ll}
\|z(x, y)-z(x, \bar{y})\|_{m} \leq Q\|y-\bar{y}\|_{r}, & \forall(x, y),(x, \bar{y}),(\bar{x}, y) \in D_{a} . \\
\|z(x, y)-z(\bar{x}, y)\|_{m} \leq\left|\int_{x}^{x} x(t) d t\right| & \\
\text { (ii) } z(x, y)=\varphi(x, y) & \forall(x, y) \in \tilde{D}_{0} .
\end{array}
$$

Romark 1: If $z \in K_{a \varphi}(P, \chi, Q)$ and $P \geq \Gamma, Q \geq \Lambda$, then for any $(x, y) \in D_{a}$ we have from the definition of $K(P, Q)$ is now satisfied with $\tau$ defined for $\alpha \in[-h, 0]$ by $\tau(\alpha)=$ $x(x+\alpha)$. In Section 3 we will introduce some additional conditions for $P, x, Q$.

We consider the quasilinear hyperbolic system of differential-functional equations in the Schauder canonic form

$$
\begin{aligned}
\sum_{j=1}^{m} & A_{i j}(x, y, z(x, y)) \\
\times & {\left[D_{x} z_{j}(x, y)+\sum_{k=1}^{T} \rho_{i k}\left(x, y, z_{x \dot{y}}\right) D_{y_{k}} z_{j}(x, y)\right] \quad(i=1, \ldots, m) } \\
& =f_{j}\left(x, y, z_{x y}\right)
\end{aligned}
$$

with initial condition

$$
z(x, y)=\varphi(x, y),(x, y) \in \cdot \widetilde{D}_{0} \cdot
$$

Any function $z \in K_{a \varphi}(P, \chi, Q)$ satisfies (2). This function is a solution of (1), (2) if it satisfies the system (1) a.e. (almost everywhere) in $D_{a}$.

If $h=0$ and $b=0$, then (1) reduces to a differential system in the Schauder canonic form. which has been studied in a large number of papers by various authors. We mention here those of L. Cesari [7, 8], P. Bassanni [2-5], M. Cinquini-Cibrario [9] and P. Pucci [12]. As a particular case of (1) we obtain a system of differential equations with a retarded argument (cf. [10]) or a few kinds of differential-integral systems (cf. for instance [6]). Differential-functional systems studied by J. Turo $[13,14]$ are also concerned in (1). More detailed description for these cases is given in Section 5 .

The aim of this paper is to prove a theorem of existence, uniqueness and continuous dependence upon Cauchy data for (1), (2). We use the method based on the Banach fixed point theorem which is close to that used in $[14]$ (see also $[8,10]$ ).

\section{Bicharacteristics}

Let $\|U\|_{m, k}=\max \left\{\sum_{j=1}^{k}\left|U_{i j}\right|: 1 \leq i \leq m\right\}$ be the norm of $U \in M(m, k), U=\left[U_{i j}\right]$.If $U \in M(m, 1)$, then we write $\|U\|_{m}$ instead of $\|U\|_{m, 1}$.

Assumption $\left(\mathrm{H}_{1}\right)$ : Suppose the following :

$1^{0} \rho: \Omega \rightarrow M(m, r)$ is such that $\rho(\because y, w):\left[0, a_{0}\right] \rightarrow M(m, r)$ is measurable for all $(y, w) \in \mathbb{R}^{r} \times K(P, Q)$, and $p(x, \cdot): \mathbb{R}^{r} \times K(P, Q) \rightarrow M(m, r)$ is continuous for a.a. $x \in\left[0, a_{0}\right]$. 
$2^{\circ}$ There are functions $n, l \in L_{1}\left(\left[0, a_{0}\right], \mathbb{R}_{+}\right)$such that for a.a. $x \in\left[0, a_{0}\right]$ and for all $(y, w),(\bar{y}, \bar{w}) \in \mathbb{R}^{r} \times K(P, Q)$ we have

$$
\begin{aligned}
& \|\rho(x, y, w)\|_{m, r} \leq n(x), \\
& \|\rho(x, y, w)-\rho(x, \bar{y}, \bar{w})\|_{m, r} \leq l(x)\left[\|y-\bar{y}\|_{r}+\|w-\bar{w}\|_{B}\right] .
\end{aligned}
$$

$3^{\circ} p, k \in(0,1)$, and $a \in\left(0, a_{0}\right]$ is sufficiently small so that $L_{a}(1+p)(1+Q) \leq p, L_{a}(1+Q)$ $\leq k$, where $L_{a}=\int_{0}^{a} l(t) d t$.

If $g=\left[g_{i j}\right] \in C\left(\Delta_{a}, M(m, r)\right)$, where $\Delta_{a}=[0, a] \times[0, a] \times \mathbb{R}^{r}$, then we write $g_{j}=$ $\left(g_{i 1}, \ldots, g_{i r}\right), j=1, \ldots, m$. By $K_{0}$ we denote the set of all functions $g \in C\left(\Delta_{a}, M(m, r)\right)$ such that for all $(x, x, y),(\xi, x, y),(\bar{\xi}, x, y),(\xi, x, y) \in \Delta_{a}$ and $j=1, \ldots, m$ we have the following :

(i) $g_{i}(x, x, y)=y$.

(ii) $\left\|g_{i}(\xi, x, y)-g_{j}(\bar{\xi}, x, y)\right\|_{r} \leq\left|\int_{\xi}^{\bar{\xi}} n(t) d t\right|$.

(iii) $\left\|g_{i}(\xi, x, y)-g_{i}(\xi, x, \bar{y})-(y-\bar{y})\right\|_{r} \leq p\left\|_{y}-\bar{y}\right\|_{r}$.

Let $\widetilde{K}_{\mathrm{O}}$ be the set of all functions $h \in C\left(\Delta_{a}, M(m, r)\right)$ defined by $h_{i}(\xi, x, y)=g_{i}(\xi, x, y)$ $-y, i=1, \ldots, m$, where $g \in K_{0}$. For $h \in \widetilde{K}_{0}$ we have the following conditions :

(i) $h_{i}(x, x, y)=0$.

(ii) $\left\|h_{i}(\xi, x, y)-h_{j}(\bar{\xi}, x, y)\right\|_{r} \leq\left|\int_{\xi}^{\bar{\xi}} n(t) d t\right|$.

(iii) $\left\|h_{j}(\xi, x, y)-h_{j}(\xi, x, \bar{y})\right\|_{r} \leq p\|y-\bar{y}\|_{r}$,

where $(x, x, y),(\xi, x, y),(\bar{\xi}, x, y),(\xi, x, \bar{y}) \in \Delta_{a}$ and $i=1, \ldots, m$. Note that the functions $h \in \tilde{K_{0}}$ are bounded. Indeed, for $(\xi, x, y) \in \Delta_{a}$ and $i=1, \ldots, m$ we have

$$
\left\|h_{i}(\xi, x, y)\right\|_{r}=\left\|h_{i}(\xi, x, y)-h_{i}(x, x, y)\right\|_{r} \leq N_{a} \text {, where } N_{a}=\int_{0}^{a} n(t) d t .
$$

It is easy to check that $\tilde{K_{0}}$ is a closed subset of the Banach space consisting of all functions $h: \Delta_{a} \rightarrow M(m, r)$ which are continuous and bounded with the norm

$$
\|h\|_{\Delta_{a}}=\sup \left\{\|h(\xi, x, y)\|_{m, r}:(\xi, x, y) \in \Delta_{a}\right\} \text {. }
$$

For any fixed $z \in K_{a \varphi}(P, \chi, Q)$ we consider the transformation $G=T_{z} g$ defined for $g$ $\varepsilon K_{0}$ by

$$
G_{i}(\xi, x, y)=y+\int_{x}^{\xi} \rho_{i}\left(t, g_{i}(t, x, y), z_{t, g_{i}(t, x, y)}\right) d t \quad\left((\xi, x, y) \in \Delta_{a} ; i=1, \ldots, m\right) .
$$

Lemma 1: If Assumption $\left(\mathrm{H}_{1}\right)$ is satisfied, then for any $z \in K_{\mathrm{a \varphi}}(P, \chi, Q)$ the transformation $T_{z}$ maps $K_{0}$ into itself and it has a unique fixed point.

The proof of this lemma is similar to that of Lemma 1 [10] and we omit the details

Romark $2:$ If $g \in K_{0}$ is a-fixed point of the transformation $T_{z}$, then for fixed $i=1, \ldots, m$ and $(x, y) \in D_{a}$ the function $g_{i}(\cdot, x, y)$ is a solution ( in the "a.e." sense) of the characte- 
ristic system of ordinary differential equations

$$
D_{t} \eta(t)=P_{i}\left(t, \eta(t), z_{t, n(t)}\right), \eta(x)=y \text {. }
$$

The functions $g_{i}$ are called bicharacteristics.

Romark 3 : If $g \in K_{0}$ is a fixed point of the transformation $T_{z}$, then for all $i=1, \ldots, m$ and $(\xi, x, y),(\xi, \bar{x}, y) \in \Delta_{a}$ we have

$$
\left\|B_{i}(\xi, x, y)-B_{i}(\xi, \bar{x}, y)\right\|_{r} \leq \lambda_{a}\left|\int_{x}^{x} n(t) d t\right| \text {. }
$$

where $\lambda_{a}=\exp \left[L_{a}(1+0)\right]$. Indeed, if $\xi \geq x$ we have

$$
\begin{aligned}
& \left\|g_{i}(\xi, x, y)-g_{i}(\xi, \bar{x}, y)\right\|_{r}
\end{aligned}
$$

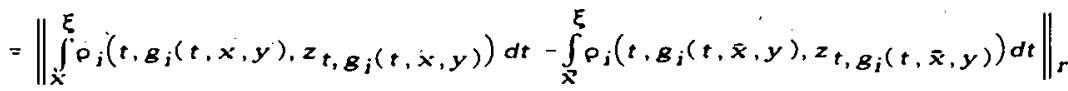

$$
\begin{aligned}
& \leq\left|\int_{x}^{\Gamma} n(t) d t\right|+\int_{x}^{\xi} n(t)(1+0)\left\|_{i} g_{j}(t, x, y)-g_{i}(t, \bar{x}, y)\right\|_{r} d t .
\end{aligned}
$$

Now, by Gronwall's inequality we obtain (4). If $\xi<x$, then by introducing a new variable $\beta$, $\xi=2 x \cdots B$, we derive the same estimate.

The fixed point of $T_{z}$ depends on a function $z \in K_{a \varphi}(P, \chi, Q)$ so we will denote it by $g[z]$.

Lemma 2 : If Assumption $\left(\mathrm{H}_{1}\right)$ is satisfied, $z, z^{\prime} \in K_{a \varphi}(P, \chi, Q)$ and if $g, g^{\prime} \in K_{O}$ are fixed points of $T_{z}, T_{z^{\prime}}$, respectively, then

$$
\|g-g \cdot\|_{\Delta_{a}} \leq L_{a} \lambda_{a}\left\|z-z^{\prime}\right\|_{\widetilde{D}_{a}}
$$

The proof of this lemma is similar to the method used in Remark 3 and it is based on Gronwall's inequality

\section{The transformation $U_{\varphi}$}

Remember that $\Omega=\left[0, a_{0}\right] \times \mathbb{R}^{r} \times K(P, Q)$ and $\Omega_{0}=\left[0, a_{0}\right] \times \mathbb{R}^{r} \times \mathbf{R}^{m}$.

Assumption $\left(\mathrm{H}_{\mathbf{2}}\right)$ : Suppose the following :

$1^{\circ} A \in C\left(\Omega_{0}, M(m, m)\right)$, and there is a constant $v>0$ such that for any $(x, y, p) \in \Omega_{0}$ we have $\operatorname{det} A(x, y, p) \geq v$.

$2^{0}$. There are constants $H, C \in \mathbb{R}_{+}$and a function $\mu \in L_{1}\left(\left[0, a_{0}\right], \mathbb{R}_{+}\right)$such that for all $(x, y, p),(x, \bar{y}, \bar{p}),(\bar{x}, y, p) \in \Omega_{0}$ we have

$$
\begin{aligned}
& \|A(x, y, p)\|_{m, m} \leq H \\
& \|A(x, y, p)-A(x, \bar{y}, \bar{p})\|_{m, m} \leq C\left[\|y-\bar{y}\|_{r}+\|p-\bar{p}\|_{m}\right] \\
& \|A(x, y, p)-A(\bar{x}, y, p)\|_{m, m} \leq\left|\int_{\lambda}^{\bar{x}} \mu(t) d t\right|
\end{aligned}
$$

Romark 4 : The above assumption implies that for any $(x, y, p) \in \Omega_{0}$ there exists an inverse matrix $A^{-1} \in C\left(\Omega_{0}, M(m, m)\right)$. There are also constants $H^{\prime}, C^{\prime} \epsilon \mathbb{R}_{+}$and a function 
$\mu^{\prime} c L_{1}\left(\left[0, a_{0}\right], R_{+}\right)$such that for all $(x, y, p),(x, \bar{y}, \bar{p}),(\bar{x}, y, p) \in \Omega_{0}$ we have

$$
\begin{aligned}
& \left\|A^{-1}(x, y, p)\right\|_{m, m} \leq H^{\prime}, \\
& \left\|A^{-1}(x, y, p)-A^{-1}(x, \bar{y}, \bar{p})\right\|_{m, m} \leq C^{\prime}\left[\|y-\bar{y}\|_{r}+\|p-\bar{p}\|_{m}\right], \\
& \left\|A^{-1}(x, y, p)-A^{-1}(\bar{x}, y, p)\right\|_{m, m} \leq\left|\int_{x}^{\bar{x}} \mu^{\prime}(t) d t\right| .
\end{aligned}
$$

Assumption $\left(\mathrm{H}_{3}\right)$ : Suppose the following :

$1^{\circ} f: \Omega \rightarrow M(m, 1)$ is such that $f(\cdot, y, w):\left[0, a_{0}\right] \rightarrow M(m, 1)$ is measurable for all $(y, w)$ $\in \mathbb{R}^{r} \times K(P, Q)$ and $f(x, \cdot): \mathbb{R}^{r} \times K(P, Q) \rightarrow M(m, 1)$ is continuous for a.a. $x \in\left[0, a_{0}\right]$.

$2^{\circ}$ There are functions $n_{1}, l_{1} \in L_{1}\left(\left[0, a_{0}\right], \mathbb{R}_{+}\right)$such that for a.a. $x \in\left[0, a_{0}\right]$ and for all $(y, w),(\bar{y}, \bar{w}) \in \mathbf{R}^{r} \times K(P, Q)$ we have

$$
\begin{aligned}
& \|f(x, y, w)\|_{m} \leq n_{1}(x) \\
& \|f(x, y, w)-f(x, \bar{y}, \bar{w})\|_{m} \leq I_{1}(x)\left[\|y-\bar{y}\|_{r}+\|w-\bar{w}\|_{B}\right] .
\end{aligned}
$$

Suppose that $(t, x, y) \in \Delta_{a}, z \in K_{a \varphi}(P, x, Q)$ and $g=g[z] \in K_{0}$ is the fixed point of $T_{z}$. Then we write

$$
\begin{aligned}
& A^{*}(t, x, y)=\left[A_{i j}\left(t, g_{i}(t, x, y), z\left(t, g_{i}(t, x, y)\right)\right)\right]_{i, j=1, \ldots, m}, \\
& \varphi^{*}(t, x, y)=\left[\varphi_{i}\left(0, g_{j}(t, x, y)\right)\right]_{i, j=1, \ldots, m}, \\
& z^{*}(t, x, y)=\left[z_{i}\left(t, g_{j}(t, x, y)\right)\right]_{i, j=1, \ldots, m}, \\
& f^{*}(t, x, y)=\left[f_{1}\left(t, g_{1}(t, x, y), z_{t, g_{1}(t, x, y)}\right), \ldots, f_{m}\left(t, g_{m}(t, x, y), z_{t, g_{m}(t, x, y)}\right)\right] \cdot .
\end{aligned}
$$

For any matrices $U=\left[U_{i j}\right], V=\left[V_{i j}\right] \in M(m, m)$ we define

$$
U * V=\left[c_{1}, \ldots, c_{m}\right]^{\top}, \text { where } c_{i}=\sum_{j=1}^{m} U_{i j} V_{j i}:(j=1, \ldots, m) .
$$

Now, for $a \in\left(0, a_{0}\right], \varphi \in J$ let the transformation $Z=U_{\varphi} z$ be defined for $z \in K_{a \varphi}(P, \chi, Q)$ by

$$
\begin{aligned}
Z(x, y)= & A^{-1}(x, y, z(x, y))\left\{A^{*}(0, x, y) * \varphi^{\bullet}(0, x, y)\right. \\
& \left.+\int_{0}^{x}\left[D_{t} A^{\bullet}(t, x, y) \cdot z \bullet(t, x, y)+f^{\bullet}(t, x, y)\right] d t\right\} \quad\left((x, y) \in D_{a}\right), \\
Z(x, y)= & \varphi(x, y) \quad\left((x, y) \in[-h, 0) \times \mathbb{R}^{r}\right) ; .
\end{aligned}
$$

Remark 5: Because the function $\Lambda$ is absolutely continuous in $x$ and Lipschitzian in $y$ and $p$, the function $z$ is absolutely continuous in $x$ and Lipschitzian in $y$, and the function $B$ is absolutely continuous in $t$, so the composite function $A^{*}$ is absolutely continuous in $t$. Then the derivative $D_{t} A^{*}$ in (6) exists a.e. in $\Delta_{a}$ and it is integrable in $t$.

Note that

$$
\begin{aligned}
& A^{-1}(x, y, z(x, y))\left[A^{\bullet}(x, x, y) \cdot \varphi^{\bullet}(x, x, y)\right] \\
& \quad=A^{-1}(x, y, z(x, y)) A(x, y, z(x, y)) \varphi(0, y)=\varphi(0, y) .
\end{aligned}
$$


By adding and subtracting $\varphi(0, y)$ in $(6)$ we obtain

$$
\begin{aligned}
Z(x, y)= & \varphi(0, y)+A^{-1}(x, y, z(x, y))\left\{A^{*}(0, x, y) * \varphi^{*}(0, x, y)\right. \\
& -A^{*}(x, x, y) \cdot \varphi^{*}(x, x, y) \\
& \left.+\int_{0}^{x}\left[D_{t} A^{*}(t, x, y) \cdot z^{*}(t, x, y)+f^{*}(t, x, y)\right] d t\right\} .
\end{aligned}
$$

Then by using

$$
A^{*}(x, x, y)-A^{*}(0, x, y)=\int_{0}^{x} D_{t} A^{*}(t, x, y) d t
$$

we derive for $(x, y) \in D_{a}$ the relation

$$
Z(x, y)=\varphi(0, y)+A^{-1}(x, y, z(x, y))\left(\Delta_{1}(x, y)+\Delta_{2}(x, y)+\Delta_{3}(x, y)\right)
$$

with

$$
\begin{aligned}
& \Delta_{1}(x, y)=\int_{0}^{x} f^{*}(t, x, y) d t, \\
& \Delta_{2}(x, y)=A^{*}(0, x, y) \cdot[\varphi *(0, x, y)-\varphi \cdot(x, x, y)], \\
& \Delta_{3}(x, y)=\int_{0}^{x} D_{t} A^{*}(t, x, y) \cdot[z *(t, x, y)-\varphi \cdot(x, x, y)] d t .
\end{aligned}
$$

Theorem 1: Suppose that Assumptions $\left(\mathrm{H}_{1}\right)-\left(\mathrm{H}_{3}\right)$ are satisfied. Then there exist $P, Q \in \mathbb{R}_{+}, \chi \in L_{1}\left(\left[0, a_{0}\right], \mathbb{R}_{+}\right)$and $a \in\left(0, a_{0}\right]$ such that for any $\varphi \in J$ the transformation $U_{\varphi}$ maps $K_{a \varphi}(P, \chi, Q)$ into itself.

Proof : Let us choose constants $P, Q \in \mathrm{R}_{+}$such that $P>\Gamma$ and

$$
Q>\Lambda\left(1+H H^{\prime}(2+p)\right) \text {. }
$$

Furthermore, let us choose constants $R_{0}, R_{1}, R_{2}, R_{3} \in \mathbf{R}_{+}$such that

$$
R_{0}>0, R_{1}>0, R_{2}>H^{\cdot}, R_{3}>H H^{\prime} \Lambda(1-k)^{-1} \text {. }
$$

Now we define a function $\chi_{1}$ for $x \in\left[0, a_{0}\right]$ by

$$
\chi_{1}(x)=R_{0} \mu(x)+R_{1} \mu^{\prime}(x)+R_{2} n_{1}(x)+R_{3} n(x) .
$$

Let $x \in L_{1}\left(\left[0, a_{0}\right], R_{+}\right)$be any function such that $\chi(x) \geq \chi_{1}(x)$ for a.a. $x \in\left[0, a_{0}\right], x(x)$ $z \omega(x)$ for a.a. $x \in[-h, 0]$. Because $\Delta_{1}(0, y)=\Delta_{2}(0, y)=\Delta_{3}(0, y)=0$ for $y \in \mathbb{R}^{r}$, so $Z(0, y)=\varphi(0, y)$ for $y \in \mathbb{R}^{r}$. Then from the definition (8) we have

$$
Z(x, y)=\varphi(x, y), \quad(x, y) \in \tilde{D}_{0} .
$$

Thus we derive

$$
Z \in C\left(\tilde{D}_{a}, \mathbb{R}^{m}\right) \text {. }
$$

For any $a \in\left(0, a_{0}\right]$ we write

$$
N_{1 a}=\int_{0}^{a} n_{1}(t) d t, L_{1 a}=\int_{0}^{A} l_{1}(t) d t, M_{a}=\int_{0}^{a} \mu_{\varphi}(t) d t, X_{a}=\int_{0}^{a} \chi_{\varphi}(t) d t .
$$

For any $(x, y) \in D_{a}$ we have the following estimates : 


$$
\begin{aligned}
\left\|\Delta_{1}(x, y)\right\|_{m} & \leq \int_{0}^{x}\|f \cdot(t, x, y)\|_{m} d t \leq \int_{0}^{x} n_{1}(t) d t \leq N_{1 a} . \\
\left\|\Delta_{2}(x, y)\right\|_{m} & \leq \max _{1 \leq i \leq m} \sum_{j=1}^{m}\left|A_{i j}^{*}(0, x, y)\right| \Lambda\left\|g_{j}(0, x, y)-g_{i}(x, x, y)\right\|_{r} \\
& \leq \max _{1 \leq i \leq m} \sum_{j=1}^{m}\left|A_{i j}^{*}(0, x, y)\right| \Lambda \int_{0}^{x} n(t) d t \leq H \Lambda N_{a} . \\
\left\|\Delta_{3}(x, y)\right\|_{m} & \leq \int_{0}^{x} \max _{1 \leq i \leq m} \sum_{j=1}^{m}\left|D_{t} A_{j j}^{*}(t, x, y)\right|\left(X_{a}+Q N_{a}\right) d t \\
& \leq\left(M_{a}+r C(1+m Q) N_{a}+m C X_{a}\right)\left(X_{a}+Q N_{a}\right)=S_{a} .
\end{aligned}
$$

From the above estimates we derive

$$
\|Z(x, y)\|_{m} \leq \Gamma+H^{\prime}\left(N_{1 a}+H \Lambda N_{a}+S_{a}\right), \quad(x, y) \in D_{a} .
$$

If we assume a sufficiently small so that

$$
\Gamma+H^{\prime}\left(N_{1 a}+H \Lambda N_{a}+S_{a}\right) \leq P,
$$

then for $(x, y) \in D_{a}$ we have $\|Z(x, y)\|_{m} \leq P$. Furthermore, since

$$
\|Z(x, y)\|_{m}=\|\varphi(x, y)\|_{m} \leq \Gamma<P,(x, y) \in \widetilde{D}_{\mathrm{o}},
$$

we have

$$
\|Z(x, y)\|_{m} \leq P, \quad(x, y) \dot{\epsilon} \tilde{D}_{a} .
$$

For all $(x, y),(x, \bar{y}) \in D_{a}$ we have

$$
Z(x, y)-Z(x, \dot{\bar{y}})=\delta_{1}+\delta_{2}+\delta_{3}+\delta_{4}+\delta_{5},
$$

where

$$
\begin{aligned}
& \delta_{1}=\varphi(0, y)-\varphi(0, \bar{y}), \\
& \delta_{2}=\left[A^{-1}(x, y, z(x, y))-A^{-1}(x, \bar{y}, z(x, \bar{y}))\right]\left(\Delta_{1}(x, y)+\Delta_{2}(x, y)+\Delta_{3}(x, y)\right), \\
& \delta_{i}=A^{-1}(x, \bar{y}, z(x, \bar{y}))\left(\Delta_{i-2}(x, y)-\Delta_{i-2}(x, \bar{y})\right), i=3,4,5 .
\end{aligned}
$$

By using $\left(\mathrm{H}_{1}\right)-\left(\mathrm{H}_{3}\right)$ we can estimate the above terms as follows:

$$
\begin{aligned}
\left\|\delta_{1}\right\|_{m} & =\|\varphi(0, y)-\varphi(0, \bar{y})\| \leq \Lambda\|y-\bar{y}\|_{r}, \\
\left\|\delta_{2}\right\|_{m} \leq & C^{\prime}(1+Q)\left[\left\|\Delta_{1}(x, y)\right\|_{m}+\left\|\Delta_{2}(x, y)\right\|_{m}+\left\|\Delta_{3}(x, y)\right\|_{m}\right]\|y-\bar{y}\|_{r} \\
& \leq C^{\prime}(1+Q)\left(N_{1 a}+H \Lambda N_{a}{ }^{-}+S_{a}\right)\|y-\bar{y}\|_{r}, \\
\left\|\delta_{3}\right\|_{m} \leq & H^{\cdot} \int_{0}^{x}\left\|f \cdot(t, x, y)-f^{*}(t, x, \bar{y})\right\|_{m} d t \\
& \leq H^{\cdot} \int_{0}^{x} I_{1}(t)(1+Q)(1+p)\|y-\bar{y}\|_{r} d t \\
& \leq H^{\cdot}(1+Q)(1+p) L_{1 a}\|y-\bar{y}\|_{r}, \\
\left\|\delta_{4}\right\|_{m} \leq & H^{\cdot}\left\{\left\|\left[A^{*}(0, x, y)-A^{*}(0, x, \bar{y})\right] \cdot\left[\varphi^{*}(0, x, y)-\varphi^{*}(x, x, y)\right]\right\|_{m}\right. \\
& \left.+\left\|A^{*}(0, x, \bar{y}) \cdot\left[\varphi^{*}(0, x, y)-\varphi^{*}(0, x, \bar{y})-\left(\varphi^{*}(x, x, y)-\varphi^{*}(x, x, \bar{y})\right)\right]\right\|_{m}\right\}
\end{aligned}
$$




$$
\begin{aligned}
\leq H & {\left[C(1+Q)(1+p) \Lambda N_{a}+H \Lambda(2+p)\right]\|y-\bar{y}\|_{r} } \\
\left\|\delta_{5}\right\|_{m} \leq H^{\prime} & \left\{\|\left[A^{*}(x, x, y)-A^{*}(x, x, \bar{y})\right] \cdot\left[z^{*}(x, x, y)-\varphi^{*}(x, x, y)\right]\right. \\
& -\left[A^{*}(0, x, y)-A^{*}(0, x, y)\right] \cdot\left[z^{*}(0, x, y)-\varphi^{*}(x, x, y)\right] \\
& -\int_{0}^{x}\left[A^{*}(t, x, y)-A^{*}(t, x, \bar{y})\right] * D_{t} z^{*}(t, x, y) d t \|_{m} \\
& +\| \int_{0}^{x} D_{t} A^{*}(t, x, \bar{y}) *\left[\left(z^{*}(t, x, y)-z^{*}(t, x, \bar{y})\right)\right. \\
& \left.-\left(\varphi^{*}(x, x, y)-\varphi^{*}(x, x, \bar{y})\right] d t \|_{m}\right\} \\
\leq & H^{\prime}\left[C(1+Q) X_{a}+C(1+Q)(1+p) Q N_{a}+C(1+Q)(1+p)\left(X_{a}+r Q N_{a}\right)\right. \\
& \left.+\left(M_{a}+r C(1+m Q) N_{a}+m C X_{a}\right)(Q(p+1)+\Lambda)\right]\|y-\bar{y}\|_{r} .
\end{aligned}
$$

In estimating $\delta_{5}$ we have used integrating by parts. From the above estimates we derive

$$
\|Z(x, y)-Z(x, \bar{y})\|_{m} \leq\left[\Lambda\left(1+H H^{\prime}(2+p)\right)+\Sigma_{a}\right]\|y-\bar{y}\|_{s},
$$

where $\Sigma_{a} \in \mathbb{R}_{+}$is some constant such that $\lim _{a \rightarrow 0^{+}} \Sigma_{a}=0$. By force of (8) we can choose a sufficiently small so that

$$
\Lambda\left(1+H H^{\prime}(2+p)\right)+\Sigma_{a} \leq Q,
$$

and then we have $\|Z(x, y)-Z(x, y)\|_{m} \leq Q\|y-\bar{y}\|_{r}$ for $(x, y),(x, \bar{y}) \in D_{a}$. Furthermore, since $Q>\Lambda$ and $Z(x, y)=\varphi(x, y)$ for $(x, y) \in \widetilde{D}_{0}$, we obtain

$$
\|Z(x, y)-Z(x, \bar{y})\|_{m} \leq Q\|y-\bar{y}\|_{r},(x, y),(x, \bar{y}) \in \tilde{D}_{a} .
$$

For all $(x, y),(\bar{x}, y) \in D_{a}$ we have

$$
Z(x, y)-Z(\bar{x}, y)=\sigma_{1}+\sigma_{2}+\sigma_{3}+\sigma_{4} \text {, }
$$

where

$$
\begin{aligned}
& \sigma_{1}=\left[A^{-1}(x, y, z(x, y))-A^{-1}(x, y, z(\bar{x}, y))\right]\left(\Delta_{1}(x, y)+\Delta_{2}(x, y)+\Delta_{3}(x, y)\right), \\
& \sigma_{i}=A^{-1}(\bar{x}, y, z(\bar{x}, y))\left(\Delta_{j-1}(x, y)-\Delta_{i-1}(\bar{x}, y)\right), i=2,3,4 .
\end{aligned}
$$

By force of $\left(\mathrm{H}_{1}\right)-\left(\mathrm{H}_{3}\right)$ and (4) we can estimate $\sigma_{1}, \ldots, \sigma_{4}$ as follows :

$$
\begin{aligned}
\left\|\sigma_{1}\right\|_{m} \leq & \left(N_{1 a}+H \Lambda N_{a}+S_{a}\right)\left(\left|\int_{x}^{\bar{x}} \mu^{\prime}(t) d t\right|+C^{\prime}\left|\int_{x}^{\bar{x}} \chi(t) d t\right|\right) \\
\left\|\sigma_{2}\right\|_{m} \leq & H^{\prime}\left(L_{1 a}(1+Q) \lambda_{a}\left|\int_{x}^{x} n(t) d t\right|+\left|\int_{x}^{x} n_{1}(t) d t\right|\right) \\
\left\|\sigma_{3}\right\|_{m} \leq & \left(H^{\prime} C(1+Q) \lambda_{a} \Lambda N_{a}+H^{\prime} H \Lambda \lambda_{a}\right)\left|\int_{x}^{\bar{x}} n(t) d t\right| \\
\left\|\sigma_{4}\right\|_{m} \leq & \left\{H^{\prime} C(1+Q) \lambda_{a}\left(2 X_{a}+(r+1) Q N_{a}\right)\right. \\
& \left.+H^{\prime} Q \lambda_{a}\left(M_{a}+r C(1+m Q) N_{a}+m C X_{a}\right)\right\}\left|\int_{x}^{x} n(t) d t\right| \\
& +H^{\prime}\left(X_{a}+Q N_{a}\right)\left|\int_{x}^{\bar{x}}(\mu(t)+r C(1+m Q) n(t)+m C \chi(t)) d t\right|
\end{aligned}
$$


Thus we have

$$
\begin{aligned}
\|Z(x, y)-Z(\bar{x}, y)\|_{m} \leq \gamma_{1 a}\left|\int_{x}^{\bar{x}} \mu(t) d t\right|+\gamma_{2 a}\left|\int_{x}^{\bar{x}} \mu^{\prime}(t) d t\right|+H\left|\int_{x}^{\bar{x}} n_{1}(t) d t\right| \\
+\left(H H \Lambda \lambda_{a}+\gamma_{3 a}\right)\left|\int_{x}^{\bar{x}} n(t) d t\right|+\gamma_{4 a}\left|\int_{X}^{\bar{x}} \chi(t) d t\right|
\end{aligned}
$$

where $\gamma_{i a} \in \mathbb{R}_{+}$are constants such that $\lim _{a \rightarrow 0+} \gamma_{i a}=0$. By force of (9) we can choose a sufficiently small so that

$$
\begin{aligned}
& \gamma_{1 a} \leq\left(1-\gamma_{4 a}\right) R_{0}, \gamma_{2 a} \leq\left(1-\gamma_{4 a}\right) R_{1}, \\
& H^{\prime} \leq\left(1-\gamma_{4 a}\right) R_{2}, \gamma_{3 a} \leq\left(1-\gamma_{4 a}\right) R_{3}-H H \Lambda \lambda_{a} .
\end{aligned}
$$

Then from $(10)$ for $(x, y),(\bar{x}, y) \in D_{a}$ we have

$$
\begin{aligned}
& \|Z(x, y)-Z(\bar{x}, y)\|_{m} \\
& \quad \leq\left(1-\gamma_{4 a}\right) R_{0}\left|\int_{x}^{\bar{x}} \mu(t) d t\right|+\left(1-\gamma_{4 a}\right) R_{1}\left|\int_{x}^{\bar{x}} \mu^{\prime}(t) d t\right| \\
& \quad+\left(1-\gamma_{4 a}\right) R_{2}\left|\int_{x^{x}}^{\bar{x}} n_{1}(t) d t\right|+\left(1-\gamma_{4 a}\right) R_{3}\left|\int_{x}^{\bar{x}} n(t) d t\right|+\gamma_{4 a}\left|\int_{x}^{\bar{x}} \chi(t) d t\right| \\
& \quad=\left(1-\gamma_{4 a}\right)\left|\int_{x}^{\bar{x}} \chi_{1}(t) d t\right|+\gamma_{4 a}\left|\int_{x}^{\bar{x}} \chi(t) d t\right| \leq\left|\int_{x}^{\bar{x}} \chi(t) d t\right| .
\end{aligned}
$$

Since $\chi(t) \geq \omega(t)$ a.e. on $[-h, 0]$ and $Z(x, y)=\varphi(x, y)$ for $(x, y) \in \widetilde{D}_{0}$, it follows that

$$
\|Z(x, y)-Z(\bar{x}, y)\|_{m} \leq\left|\int_{x}^{\bar{x}} \chi(t) d t\right|, \quad(x, y),(\bar{x}, y) \in \tilde{D}_{a} .
$$

Therefore if we assume a sufficiently small so that the inequalities (13), (15), (17) hold, then by force of (11), (12), (14), (16), (18) the transformation $U_{\varphi}$ maps $K_{a \varphi}(P, \chi, Q)$ into itself

For a $\in\left(0, a_{0}\right]$ we define constants

$$
\begin{aligned}
G_{a}=1 & +2 H^{\prime} H+H^{\prime}\left(M_{a}+r C(1+m Q) N_{a}+m C X_{a}\right), \\
G_{1 a}^{\prime}= & C^{\prime}\left(N_{1 a}+H \Lambda N_{a}+S_{a}\right), \\
G_{2 a}^{\prime}= & H^{\prime} L_{1 a}\left(1+(1+Q) \lambda_{a} L_{a}\right), \\
G_{3 a}^{\prime}= & H^{\prime} \Lambda\left[H \lambda_{a} L_{a}+C N_{a}\left(1+(1+Q) \lambda_{a} N_{a}\right)\right], \\
G_{4 a}^{\prime}= & H^{\prime}\left[C X_{a}+C\left(X_{a}+Q N_{a}(1+r)\right)\left(1+(1+Q) \lambda_{a} L_{a}\right)\right. \\
& \left.+\left(M_{a}+r C(1+m Q) N_{a}+m C X_{a}\right)\left(1+Q \lambda_{a} L_{a}\right)\right] .
\end{aligned}
$$

Lemma 3 : If Assumptions $\left(\mathrm{H}_{1}\right)-\left(\mathrm{H}_{3}\right)$ are satisfied, then for all $a \in\left(0, a_{0}\right], \varphi, \varphi^{\prime} \in J$, and $z \in K_{a \varphi}(P, \chi, Q), z^{\prime} \in K_{a \varphi^{\prime}}(P, \chi, Q)$ we have

$$
\left\|U_{\varphi} z-U_{\varphi} \cdot z^{\prime}\right\|_{D_{a}} \leq G_{a}\left\|\varphi-\varphi^{\prime}\right\|_{D_{Q}}+G_{a}^{\prime}\left\|_{z}-z^{\prime}\right\|_{D_{a}}, G_{a}^{\prime}=\sum_{i=1}^{1} G_{i a}^{\prime} .
$$

Proof : Let $\varphi, \varphi^{\prime} \in J, z \in K_{\mathbf{a} \varphi}(P, \chi, Q), z^{\prime} \in K_{a \varphi^{\prime}}(P, \chi, Q)$ and let $g, g^{\circ} \in K_{0}$ be the fixed points of $T_{z}, T_{z^{\prime}}$, respectively. Then for any $(x, y) \in D_{a}$ we have

$$
\left(U_{\varphi} z\right)(x, y)-\left(U_{\varphi} \cdot z^{\prime}\right)(x ; y)=\varepsilon_{0}+\varepsilon_{1}+\varepsilon_{2}+\varepsilon_{3}+\varepsilon_{4},
$$


where $\varepsilon_{0}=\varphi(0, y)-\varphi^{\prime}(0, y)$ and

$$
\begin{aligned}
& \varepsilon_{1}=\left[A^{-1}(x, y, z(x, y))-A^{-1}\left(x, y, z^{\prime}(x, y)\right)\right]\left(\Delta_{1}(x, y)+\Delta_{2}(x, y)+\Delta_{3}(x, y)\right), \\
& \varepsilon_{i}=A^{-1}\left(x, y, z^{\prime}(x, y)\right)\left(\Delta_{i-1}(x, y)-\Delta_{i-1}^{\prime}(x, y)\right), \quad i=2,3,4,
\end{aligned}
$$

and formulas for $\Delta_{j-1}^{\prime}$ arise from (7) with functions $\varphi, z, g$ replaced by $\varphi^{\prime}, z^{\prime}, g^{\prime}$, respectively. Using the same methods as in the proof of Theorem 1 we can estimate the above terms as follows :

$$
\begin{aligned}
& \left\|\varepsilon_{0}\right\|_{m} \leq\left\|\varphi-\varphi^{\prime}\right\|_{D_{0}}, \quad\left\|\varepsilon_{1}\right\|_{m} \leq G_{1 a}^{\prime}\left\|z-z^{\prime}\right\|_{D_{a}}, \\
& \left\|\varepsilon_{2}\right\|_{m} \leq H^{\prime} \int_{0}^{x} l_{1}(t)\left((1+Q)\left\|_{g}-g^{\prime}\right\|_{\Delta_{a}}+\left\|z-z^{\prime}\right\|_{D_{a}}\right) d t \leq G_{2 a}^{\prime}\left\|z-z^{\prime}\right\|_{D_{a}} \text {, } \\
& \left\|\varepsilon_{3}\right\|_{m} \leq H^{\prime}\left\{\|[A \uparrow 0, x, y)-A^{* *}(0, x, y)\right] \cdot\left[\varphi^{*}(0, x, y)-\varphi^{*}(x, x, y)\right] \|_{m} \\
& \left.+\left\|A^{\cdot}(0, x, y) \cdot\left[\left(\varphi^{\bullet}(0, x, y)-\varphi^{\prime \bullet}(0, x, y)\right)-\left(\varphi^{*}(x, x, y)-\varphi^{*}(x, x, y)\right)\right]\right\|_{m}\right\} \\
& \leq 2 H^{\prime} H\left\|\varphi-\varphi^{\prime}\right\|_{\tilde{D}}+G_{3 a}^{\prime}\left\|z-z^{\prime}\right\|_{\widetilde{D}} a^{\prime} \\
& \left\|\varepsilon_{4}\right\|_{m} \leq H^{\prime}\left\{\|\left[A^{\bullet}(x, x, y)-A^{*}(x, x, y)\right] \cdot\left[z^{*}(x, x, y)-\varphi^{*}(x, x, y)\right]\right. \\
& \text { - }\left[A^{*}(0, x, y)-A^{*}(0, x, y)\right] \cdot\left[z^{*}(0, x, y)-\varphi^{*}(x, x, y)\right] \\
& -\int_{0}^{x}\left[A^{*}(t, x, y)-A^{*}(t, x, y)\right] \cdot D_{t} z^{*}(t, x, y) d t \|_{m} \\
& +\left\|\int_{0}^{x} D_{t} A^{\prime}(t, x, y) \cdot\left[\left(z^{*}(t, x, y)-z^{*}(t, x, y)\right)-\left(\varphi^{*}(x, x, y)-\varphi^{\prime *}(x, x, y)\right)\right] d t\right\|_{m} \\
& s H^{\prime}\left(M_{a}+r C(1+m Q) N_{a}+m C X_{a}\right)\left\|\varphi-\varphi^{\bullet}\right\|_{D_{0}}+G_{4 a}^{\prime}\left\|z-z^{\prime}\right\|_{D_{a}}
\end{aligned}
$$

From the above estimates we obtain

$$
\left\|\left(U_{\varphi} z\right)(x ; y)-\left(U_{\varphi^{\prime}} z^{\prime}\right)(x, y)\right\|_{m} \leq G_{a}\left\|\varphi-\varphi^{\prime}\right\|_{\tilde{D}_{0}}+G_{a}^{\prime}\left\|z-z^{\prime}\right\|_{D_{a}},(x, y) \in D_{a} .
$$

Because $G_{a} \geq 1, G_{a}^{\prime} \geq 0$, so the same inequality holds for $(x, y) \in \widetilde{D}_{0}$

\section{The main theorem}

We are now in a position to show a theorem on the exitence, uniqueness and continuous dependence upon the Cauchy data for the problem (1), (2).

Theorem 2 : Suppose that the Assumptions $\left(\mathrm{H}_{1}\right)-\left(\mathrm{H}_{3}\right)$ are satisfied. Then for any $\varphi$ $\in J$ and for $a \in\left(0, a_{0}\right]$ sufficiently small there exists a function $z \in K_{a \varphi}(P, \chi, Q)$ being a unique solution of the problem (1), (2) in the class $K_{a \varphi}(P, \chi, Q)$. Furthermore, if $z, z^{\prime}$ are solutions of (1), (2) with initial functions $\varphi, \varphi^{\prime} \in J$, respectively, then

$$
\left\|z-z^{\prime}\right\|_{D_{a}^{\prime}} \leq G_{a}(1-k)^{-1}\left\|\varphi-\varphi^{\prime}\right\|_{D_{0}^{\prime}}
$$

Proof: Suppose that $a \in\left(0, a_{0}\right]$ is sufficiently small so that there are satisfied the inequalities (13), (15), (17) and

$$
G_{a}^{\prime} \leq k \text {. }
$$

Then for any $\varphi \in J$ the transformation $U_{\varphi}$ maps $K_{a \varphi}(P, \chi, Q)$ into itself and by force of 
(19), (21) we have

$$
\left\|U_{\varphi} z-U_{\varphi} z^{\prime}\right\|_{D_{\mathbf{a}}} \leq k\left\|_{z}-z^{\prime}\right\|_{\boldsymbol{D}_{\mathbf{a}}} \text { for } z, z^{\prime} \in K_{\mathbf{a}}(P, \chi, Q) \text {. }
$$

Thus the transformation $U_{\varphi}$ has a fixed point $z=z[\varphi] \in K_{a \varphi}(P, \chi, Q)$. We see at once that $z$ satisfies (2). Let us prove that $z$ satisfies (1) a.e. in $D_{a}$. For any $(x, y) \in D_{a}$ we have

$$
\begin{aligned}
z(x, y)= & A^{-1}(x, y, z(x, y))\left\{A^{*}(0, x, y) \cdot \varphi^{*}(0, x, y)\right. \\
& \left.+\int_{0}^{x}\left[D, A^{*}(t, x, y) \cdot z^{*}(t, x, y)+f^{*}(t, x, y)\right] d t\right\} .
\end{aligned}
$$

From this, by integration by parts we obtain

$$
\begin{aligned}
A(x, y, z(x, y)) z(x, y)= & A^{*}(0, x, y) * \varphi^{*}(0, x, y) \\
& +\left[A^{*}(x, x, y) * z^{*}(x, x, y)-A^{*}(0, x, y) * z^{*}(0, x, y)\right] \\
& +\int_{0}^{x}\left[-A^{*}(t, x, y) * D_{t} z^{*}(t, x, y)+f^{*}(t, x, y)\right] d t,
\end{aligned}
$$

which yields

$$
\int_{0}^{x}\left[-A^{\bullet}(t, x, y) \cdot D_{t} z^{\bullet}(t, x, y)+f^{\cdot}(t, x, y)\right] d t=0,(x, y) \in D_{a} .
$$

For $i s i s m$ the function $g_{j}=g_{j}[\dot{z}]$ is a unique solution of the Cauchy problem (3) and therefore using the groupal property we obtain (cf. [8])

$$
y=g_{j}(x, 0, \eta) \text { if and only if } \eta=g_{i}(0, x, y) .
$$

For a fixed $x \in[0, a]$ the above relation represents a one-to-one transformation of the space $\mathbb{R}^{r}$ into itself. Furthermore, it is easy to prove that this transformation together with its inverse is Lipschitz continuous. If $1 \leq i \leq m$, then writing (22) by co-ordinates, using the transformation $y=g_{j}(x, 0, \eta)$ and differentiating the resulting relation with respect to $x$ we derive

$$
\begin{aligned}
& \sum_{j=1}^{m} A_{i j}\left(x, g_{i}(x, 0, \eta), z\left(x, g_{i}(x, 0, \eta)\right)\right)\left[D_{x} z_{j}\left(x, g_{i}(x, 0, \eta)\right)\right. \\
& \left.+\sum_{k=1}^{r} \rho_{i k}\left(x, g_{i}(x, 0, \eta), z_{x, g_{j}}(x, 0, \eta)\right) D_{y_{k}} z_{j}\left(x, g_{i}(x, 0, \eta)\right)\right] \\
& =f_{j}\left(x, g_{j}(x, 0, \eta), z_{x, g_{j}(x, 0, \eta)}\right) \text { for a.a. }(x, \eta) \in D_{a} \text {. }
\end{aligned}
$$

By using the transformation $\eta=g_{i}(0, x, y)$, which preserves the sets of measure zero, we obtain that (1) is satisfied a.e. in $D_{a}$.

In order to prove that the solution $z$ is unique, suppose that a certain $z \in K_{a \varphi}(P, \chi, Q)$ satisfies (1) a.e. in $D_{a}$. It is easy to see that $A^{*}(t, x, y) \cdot D_{t} \widetilde{z}^{*}(t, x, y)=f^{*}(t, x, y)$ for a.a. $(t, x, y) \in \Delta_{a}$. Integrating this relation by parts we obtain

$$
\begin{aligned}
\widetilde{z}(x, y)= & A^{-1}(x, y, \widetilde{z}(x, y))\left\{A^{*}(0, x, y) \cdot \varphi^{\bullet}(0, x, y)\right. \\
& \left.+\int_{0}^{x}\left[D_{t} A^{*}(t, x, y) \cdot \widetilde{z}^{*}(t, x, y)+f^{\bullet}(t, x, y)\right] d t\right\},(x, y) \in D_{a} .
\end{aligned}
$$

Thus the function $\tilde{z}$ satisfies a fixed point equation for the transformation $U_{\varphi}$. A solution of this equation is unique so $\widetilde{z}=z$.

By force of (19), (21) we have for $z=z[\varphi] \in K_{a \varphi}(P, \chi, Q), z^{\prime}=z\left[\varphi^{\prime}\right] \in K_{a \varphi} \cdot(P, \chi, Q)$ the inequality $\left\|z-z^{\prime}\right\|_{B_{a}} \leq G_{a}\left\|\varphi-\varphi^{\prime}\right\|_{B_{0}}+k\left\|z-z^{\prime}\right\|_{B_{a}}$, from which we obtain (20) 


\section{Examples}

As a particular case of (1), (2) we obtain the initial problem for the quasilinear hyperbolic system of partial differential equations with a retarded argument

$$
\begin{aligned}
& \sum_{j=1}^{m} A_{j j}(x, y, z(x, y))\left[D_{x} z_{j}(x, y)\right. \\
&\left.+\sum_{k=1}^{r} \tilde{\rho}_{i k}(x, y, z(x, y), z(\lambda(x), \psi(x, y))) D_{y_{k}} z_{j}(x, y)\right] \\
&= f_{i}(x, y, z(x, y), z(\lambda(x), \psi(x, y))), i=1, \ldots, m, \\
& z(x, y)=\varphi(x, y),(x, y) \in \tilde{D}_{0},
\end{aligned}
$$

where $\psi(x, y)=\left(\psi_{1}(x, y), \ldots, \psi_{r}(x, y)\right)$.

Assumption $\left(\mathrm{H}_{4}\right)$ : Suppose the following :

$1^{\circ} \tilde{f}:\left[0, a_{0}\right] \times \mathbb{R}^{r} \times \mathbb{R}^{m} \times \mathbf{R}^{m} \rightarrow M(m, 1)$ and $\tilde{\rho}:\left[0, a_{0}\right] \times \mathbb{R}^{r} \times \mathbf{R}^{m} \times \mathbb{R}^{m} \rightarrow M(m, r)$ are functions such that $\tilde{f}(\cdot, y, p, q)$ and $\tilde{\rho}(\cdot, y, p, q)$ are measurable for all $(y, p, q) \in \mathbb{R}^{r} \times \mathbb{R}^{m}$ $\times \mathbb{R}^{m}$, and $\tilde{f(x, \cdot)}, \tilde{p}(x, \cdot)$ are continuous for a.a. $x \in\left[0, a_{0}\right]$.

$2^{\circ}$ There are functions $\widetilde{n}, \widetilde{n}_{1}, \widetilde{l}, \tilde{l}_{1} \in L_{1}\left(\left[0, a_{0}\right] \times \mathbb{R}_{+}\right)$such that for a.a. $x \in\left[0, a_{0}\right]$ and for all $(y, p, q),(\bar{y}, \bar{p}, \bar{q}) \in \mathbb{R}^{r} \times \mathbb{R}^{m} \times \mathbb{R}^{m}$ we have

$$
\begin{aligned}
& \|\tilde{\rho}(x, y, p, q)\|_{m, r} \leq \tilde{n}(x),\|\tilde{f}(x, y, p, q)\|_{m} \leq \tilde{n}_{1}(x) . \\
& \|\tilde{\rho}(x, y, p, q)-\tilde{p}(x, y, \bar{p}, \bar{q})\|_{m, r} \leq \tilde{l}(x)\left[\|y-\bar{y}\|_{r}+\|p-\bar{p}\|_{m}+\|q-\ddot{q}\|_{m}\right], \\
& \|\tilde{f}(x, y, p, q)-\tilde{f}(x, \bar{y}, \bar{p}, \bar{q})\|_{m} \leq \tilde{l}_{1}(x)\left[\|y-\bar{y}\|_{s}+\|p-\bar{p}\|_{m}+\|q-\bar{q}\|_{m}\right] .
\end{aligned}
$$

\section{Lemma 5 : Suppose that}

$1^{\circ}$ Assumptions $\left(\mathrm{H}_{2}\right),\left(\mathrm{H}_{4}\right)$ are satisfied,

$2^{\circ} \lambda:\left[0, a_{0}\right] \rightarrow \mathbb{R}^{r}$ is measurable and $-h \leq \lambda(x) \leq x$ for a.a. $x \in\left[0, a_{0}\right]$,

$3^{\circ} \psi(\cdot, y):\left[0, a_{0}\right] \rightarrow \mathbb{R}^{r}$ is measurable for every $y \in \mathbb{R}^{r}$ and there is a $E \in \mathbb{R}_{+}$such that $\|\psi(x, y)-\psi(x, \bar{y})\|_{r} \leq E\|y-\bar{y}\|_{r},\|\psi(x, y)-y\|_{r} \leq b$ for all $y, \bar{y} \in \mathbb{R}^{r}$ and a.a. $x \in\left[0, a_{0}\right]$.

Then the functions $\rho: \Omega \rightarrow M(m, r), f: \Omega \rightarrow M(m, 1)$ defined by

$$
\begin{aligned}
& \rho(x, y, w)=\tilde{\rho}(x, y, w(0,0), w(\lambda(x)-x, \psi(x, y)-y)), \\
& f(x, y, w)=\tilde{f}(x, y, w(0,0), w(\lambda(x)-x, \psi(x, y)-y)),
\end{aligned}
$$

satisfy Assumptions $\left(\mathrm{H}_{1}\right),\left(\mathrm{H}_{3}\right)$ with $n=\tilde{n}, n_{1}=\tilde{n}_{1}, l=\tilde{l}(Q E+Q+2), l_{1}=\tilde{l}_{1}(Q E+Q+2)$.

We omit the simple proof of this lemma

Romark 5 : In the assumptions of Lemma 5 we may drop the condition $\|\Psi(x, y)-y\|_{r}$ $s b$ for all $y \in \mathbb{R}^{r}$ and for a.a. $x \in\left[0, a_{0}\right]$, if $b=+\infty$.

As another example we consider the Cauchy problem for the system of partial differential-integral equations

$$
\begin{aligned}
\sum_{j=1}^{m} A_{i j}(x, y, z(x, y))\left[D_{x} z_{j}(x, y)\right. \\
\left.\quad+\sum_{k=1}^{r} \tilde{\rho_{i k}}\left(x, y, z(x, y), \int_{\lambda(x, y)}^{\psi(x, y)} z(s, t) K(s, t, x, y) d s d t\right) D_{y_{k}} z_{j}(x, y)\right]
\end{aligned}
$$




$$
\begin{aligned}
& =f_{i}\left(x, y, z(x, y), \int_{\lambda(x, y)}^{\psi(x, y)} z(s, t) K(s, t, x, y) d s d t\right), i=1, \ldots, m, \\
& z(x, y)=\varphi(x, y),(x, y) \in \widetilde{D}_{0},
\end{aligned}
$$

where $\lambda=\left(\lambda_{0}, \ldots, \lambda_{r}\right), \psi=\left(\psi_{0}, \ldots, \psi_{r}\right), K=\left[K_{i j}\right]_{i, j=1, \ldots, m}$.

\section{Assumption ( $\mathrm{H}_{5}$ ) : Suppose the following :}

$1^{\circ} \lambda(\cdot, y), \psi(\cdot, y):\left[0, a_{0}\right] \rightarrow \mathbb{R}^{1+r}$ are measurable for every $y \in \mathbb{R}^{r}$, and

$-h \leq \lambda_{0}(x, y) \leq x,-h \leq \psi_{0}(x, y) \leq x$

$\|\bar{\lambda}(x, y)-y\|_{r} \leq b,\|\bar{\psi}(x, y)-y\|_{r} \leq b$

$$
,(x, y) \in D_{a_{0}}
$$

where $\bar{\lambda}=\left(\lambda_{1}, \ldots, \lambda_{r}\right), \bar{\psi}=\left(\psi_{1}, \ldots, \psi_{r}\right)$.

$2^{\circ}$ There are constants $d, \tilde{d}, g \in R_{+}$such that for all $(x, y),(x, \bar{y}) \in D_{a_{0}}$ we have

$\|\lambda(x, y)-\lambda(x, \bar{y})\|_{1+r} \leq d\|y-\bar{y}\|_{r}, \quad\|\psi(x, y)-\psi(x, \bar{y})\|_{1+r} \leq \tilde{d}\|y-\bar{y}\|_{r}$,

$\prod_{j=0}^{k}\left|\psi_{j}(x, y)-\lambda_{j}(x, y)\right| \leq g, \prod_{j=k}^{r}\left|\psi_{j}(x, y)-\lambda_{j}(x, y)\right| \leq g, k=0, \ldots, r$.

$3^{\circ} K(\cdot, y):\left[0, a_{0}\right] \times \mathbb{R}^{r} \times\left[0, a_{0}\right] \rightarrow M(m, m)$ is measurable for every $y \in \mathbb{R}^{r}$.

$4^{0}$ There are constants $h_{0}, h_{1} \in \mathbb{R}_{+}$such that for all $(s, t, x, y),(s, t, x, \bar{y}) \in\left[0, a_{0}\right] \times \mathbb{R}^{r}$ $\times\left[0, a_{0}\right] \times \mathbb{R}^{r}$ we have

$$
\|K(s, t, x, y)\|_{m, m} \leq h_{0} \quad,\|K(s, t, x, y)-K(s, t, x, \bar{y})\|_{m, m} \leq h_{1}\|y-\bar{y}\|_{s} .
$$

Lemma 6: Suppose that the Assumptuions $\left(\mathrm{H}_{2}\right),\left(\mathrm{H}_{4}\right),\left(\mathrm{H}_{5}\right)$ are satisfied. Then the functions $\rho: \Omega \rightarrow M(m, r), f: \Omega \rightarrow M(m, 1)$ defined by

$$
\begin{aligned}
\rho(x, y, w) & =\tilde{\rho}\left(x, y, w(0,0), \int_{\lambda(x, y)}^{\psi(x, y)} w(s-x, t-y) K(s, t, x, y) d s d t\right), \\
f(x, y, w) & =\tilde{f}\left(x, y, w(0,0), \int_{\lambda(x, y)}^{\psi(x, y)} w(s-x, t-y) K(s, t, x, y) d s d t\right),
\end{aligned}
$$

satisfy Assumptions $\left(\mathrm{H}_{1}\right),\left(\mathrm{H}_{3}\right)$ with $n=\widetilde{n}, n_{1}=\widetilde{n}_{1}$, and

$$
\begin{aligned}
& l=\tilde{l}\left[1+g\left(h_{0}+h_{0} Q+h_{1} P\right)+(d+\widetilde{d}) h_{0} P\left(2 g+(r-1) g^{2}\right)\right], \\
& l_{1}=\tilde{l}_{1}\left[1+g\left(h_{0}+h_{0} Q+h_{1} P\right)+(d+\widetilde{d}) h_{0} P\left(2 g+(r-1) g^{2}\right)\right] .
\end{aligned}
$$

We omit the proof of this lemma and we can make an analogous remark like in Lemma 5 for the case $b=+\infty$

Finally, we consider the system which has been studied by J.Turo [14] (cf. also [13])

$$
\begin{aligned}
\sum_{j=1}^{m} A_{i j}(x, y, z(x, y)) & {\left[D_{x} z_{j}(x, y)\right.} \\
& \left.+\sum_{k=1}^{r} \tilde{P}_{i k}(x, y, z(x, y),(V z)(x, y)) D_{y_{k}} z_{j}(x, y)\right] \\
= & \tilde{f_{i}}(x, y, z(x, y),(V z)(x, y)), i=1, \ldots, m, \\
z(x, y) & =\varphi(x, y),(x, y) \in \tilde{D}_{0},
\end{aligned}
$$

where $V: K(P, Q) \rightarrow K$, and $K$ is the set of all functions $z: D_{B} \rightarrow \mathbb{R}^{m}$ such that $z(\cdot, y)$ : 
$\left[0, a_{0}\right] \rightarrow \mathbb{R}^{m}$ is measurable for all $y \in \mathbb{R}^{r}$ and $z(x, \cdot): \mathbb{R}^{r} \rightarrow \mathbb{R}^{m}$ is continuous for a.a. $x \in$ $\left[0, a_{0}\right]$. Suppose that $V$ is a continuous operator of the Volterra type. If $\varphi \in J$, then the function $\widetilde{\varphi} \in C\left([-\tilde{h}, 0] \times \mathbb{R}^{r}, \mathbb{R}^{m}\right), \widetilde{h}=h+a_{0}$, is given by $\widetilde{\varphi}(x, y)=\varphi(x, y)$ for $(x, y) \in D_{0}$ and $\widetilde{\varphi}(x, y)=\varphi(-h, y)$ for $(x, y) \in[-\tilde{h},-h) \times \mathbb{R}^{r}$. We introduce the operator

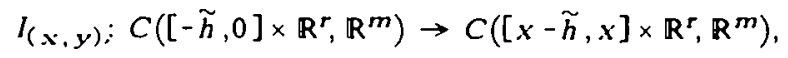

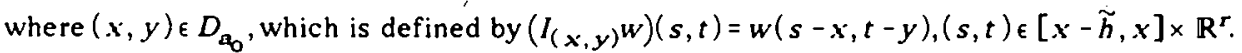
Finally, suppose that

$$
\begin{aligned}
& \rho(x, y, w)=\widetilde{\rho}\left(x, y, w(0,0),\left(V\left(I_{(x, y)} w\right)\right)(x, y)\right), \\
& f(x, y, w)=\widetilde{f}\left(x, y, w(0,0),\left(V \left(I_{\left.\left.\left.(x, y)^{w}\right)\right)(x, y)\right) .}\right.\right.\right.
\end{aligned}
$$

Now we have the problem (24), (25) obtained as a particular case of (1),(2), with $h$ and $\varphi$ replaced by $\tilde{h}$ and $\widetilde{\varphi}$, respectively, and with $b=+\infty$.

\section{REFERENCES}

[1] BASSANINI, P.: A nonlinear hyperbolic problem arising from a question of nonlinear optics. ZAMP 27 (1976), 409 - 422.

[2] BASSANINI, P.: Jteraive methods for guasilinear hyperbolic systems in the first canonic form. Appl. Anal. 12 (1981), 10s 117.

[3] BASSANINI, P.: Iterative methods for quasilinear hyperbolic systems. Boll. Un. Mat. Ital. 1-B(1982) 6, 225 - 250.

[4] BASSANINI, P.: Su una reconte dimosirazjone cirza il problema di Cauchy per sisto mi quasi lineari iperbolici. Boll. Un. Mat. Ital. 13-B (1976) 5, 322 - 335.

[S] BAssanini, P.: The problem of Graffi-Cesari. Proc. Int. Conf. Nonlin. Phen. Math. Sci. Arlington (USA) 1980. Academic Press 1982,87-101.

[6] BASSANINI, P., and M. C. SALVATORI: Ul problema ai limiti per sistemi integrodifferenziali non lineari di tipo iperbolico. Boll. Un. Mat. Ital. 18-B (1981) 5, 785-798.

[7] CESARI. L.: A boundary value problm for quasilinear hyperbolic systems. Riv. Mat. Univ. Parma 3(1974), 107 - 131.

[8] CESARI, L.: A boundary value problem for quasilinear hyperbolic systems in the Schauders canonic form. Ann. Sc. Norm. Sup. Pisa 1 (1974) 4, $311-358$.

[9] CINQUINI-CIBRARIO, M.: Teoremi di esistenza per sistemi di equazioni quasilineari a derivate parziali in piu variabili independenti. Ann. Mat. Pura Appl. 75 (1967), 146.

L10J KAMONT, Z, and J. TURO: On the Cauchy problem for quasilinear hyperbolic system of partial differential equations with a retarded argument. Boll. Un. Mat. Ital. 4-B (1985) 6, $901-916$.

[11] KAMONT, Z., and J. TURO: On the Cauchy problem for quasilinear hyperbolic systems with a retarded argument. Ant. Mat. Pura Appl. 143(1986), 235 - 246.

[12] PUCCl, P.: Problemi ai limiti per sistemi di equazioni iperboliche. Boll. Un. Mat. Ital. 16-B(1979) S, $119-128$.

[13] TURO, J.: A boundary value problem for quasilinear hyperbolic systems of hereditary partial differential equations. Atti Sem. Mat. Fis. Univ. Modena 34(1985-86), $15-34$.

[14] TURO, J.: On some class of quasilinear hyperbolic systems of partial differentialfunctional equations of the first order. Czech. Math. J. 36(1986), 185 - 197.

Received 07.02.1989, in revised form 02.07.1990

Author's address :

Dr. Tomasz Cztapinski

Institute of Mathematics of the University of Gdansk ul. Wita Stwosza 57

P - 80-952 Gdansk 